\title{
Gestión social y desarrollo sostenible en América Latina y el Caribe (2011-2021)
}

\section{Dante Hilario Salas Vizcarra \\ Dante111_@hotmail.com}

Universidad Cesar Vallejo Campus Lima Norte

Lima - Perú

\section{RESUMEN}

La convivencia entre los seres humanos y la naturaleza nos ha enseñado que al destruir el medio ambiente nos condenamos a nosotros mismos, en ese entender la Gestión Social (GS) promueve la vinculación efectiva de la comunidad en los proyectos sociales ambientales, es decir mejora la sostenibilidad del medio ambiente. En esta línea, el objetivo del presente trabajo fue analizar las técnicas y prácticas respecto de la Gestión Social y desarrollo sostenible en América Latina y el Caribe. Para ello fueron analizados 10 artículos científicos en español de las siguientes revistas: Scielo, Ebsco, Dialnet y Redalyc. Para el presente análisis, se tuvo en consideración criterios de inclusión y exclusión; se trabajó con los artículos publicados en español entre el año 2011 a 2021. Se concluye que de acuerdo a los resultados Ecuador es el país donde se concentra la mayor cantidad de investigaciones sobre enfoque cuantitativo y encuestas aplicados a directivos, y seguido de Colombia donde se encuentra en un estado más preliminar de desarrollo.

Palabras clave: medioambiente; proyectos sociales; programas sociales. 


\title{
Social management and sustainable development in Latin America and the Caribbean (2011-2021)
}

\begin{abstract}
The coexistence between human beings and nature has taught us that by destroying the environment we condemn ourselves, in that understanding Social Management (GS) promotes the effective linkage of the community in environmental social projects, that is, it improves the environmental sustainability. In this line, the objective of this work was to analyze the techniques and practices regarding Social Management and sustainable development in Latin America and the Caribbean. For this, 10 scientific articles in Spanish from the following journals were analyzed: Scielo, Ebsco, Dialnet and Redalyc. For the present analysis, inclusion and exclusion criteria were considered; We worked with articles published in Spanish between 2011 and 2021. It is concluded that according to the results, Ecuador is the country where the largest amount of research on a quantitative approach and surveys applied to executives is concentrated, and followed by Colombia where it is It is in a more preliminary stage of development.
\end{abstract}

Keywords: environment; social projects; social programs.

Artículo recibido: 20 diciembre. 2021 Aceptado para publicación: 10 enero 2022 Correspondencia: Dante111_@hotmail.com Conflictos de Interés: Ninguna que declarar 


\section{INTRODUCCIÓN}

Nuestras acciones necesitan ser reorientadas para fortalecer capacidades que nos permitan gestionar los territorios, nuestras actividades y nuestra relación con el medio natural bajo un enfoque estratégico en el que los ejes centrales del desarrollo sostenible son la gobernanza (estándares, coordinación interinstitucional, público, alianzas privadas, vinculación ciudadana, ordenamiento territorial), la preferencia a soluciones basadas en la naturaleza y la relación ciencia-tecnología-empoderamiento social (Moreno et al., 2019). La concretización de la gestión social se realiza a través de programas sociales, como imagen dinámica estructurada y una forma de acción para la interacción coordinada entre los cuerpos de poder, que permite la solución de importantes problemas sociales de acuerdo a su compromiso de recursos, ejecutores y plazos para la implementación de un conjunto de tareas y medidas (Terziev, 2018)

Existen muchos problemas ambientales en el mundo, ocasionado por la población como se mencionan: deforestación, desertificación, erosión y agotamiento de suelos, contaminación agrícola, industrial y doméstica, acumulación de residuos sólidos, sequías e inundaciones consideradas de alta prioridad para América Latina y pueden ser discutibles desde perspectiva de cada Estado en particular (Miyazaki et al., 2020; Zacarias et al., 2020). Es significativo marcar la divergencia entre el problema ambiental de la Región Latinoamericana y las causas sociales, económicas, políticas y culturales que los causan ante esta real y trágica situación, por ejemplo tenemos en Perú la contaminación local producido por la minería artesanal, dedicados a la extracción de oro de manera ilegal con importantes consecuencias ambientales como destrucción de vegetación y suelo, y liberación irresponsable de mercurio a las aguas al medio ambiente, que operan sin permisos alguno (OCDE, 2016)

En este contexto viendo la problemática, se plantean acciones para la gestión social (GS) del conocimiento medioambiental en favor de la toma de conciencia participativa de la ciudadanía, y solucionar problemas que aquejan el desarrollo de la sociedad, de esta forma elevar la conciencia, el conocimiento ambiental de los pobladores; y esto se logra gracias al trabajo en unidad para reducir los niveles de contaminación, tener una naturaleza sana y limpia (Varona, 2018). La GS de los proyectos adquiere una relevancia importante, ya que tiene implicaciones directas sobre la seguridad, la sostenibilidad y en lo social, en el ámbito de los proyectos cubre un aspecto muy amplio en el desarrollo local, regional o 
nacional y sus implicaciones socioeconómicas (Mendieta et al., 2018; Hernández, 2017; BID, 2017)

En este sentido, la GS es el comportamiento de las organizaciones con el interés bajo los lineamientos de sus valores; ayudando a la solución de problemas sociales, económicos, educativos y ambientales, involucrando a las empresas con la responsabilidad (Chávez et al., 2018; Muñoz, 2017). Los programas sociales juegan un papel importante en los métodos de gestión social empleados y las necesidades directas, y responsabilidades requeridas de un administrador social, que difieren significativamente de un generalista a un enfoque de beneficiarios específicos (Abdelfattah, 2018). Además la GS busca un camino para la autonomía del individuo a través de la participación en las decisiones en las que están involucrados, en donde la ciudadanía tiene derecho a opinar sin restricciones (Ariel et al., 2019; Quintana et al., 2018).

Es pertinente indicar, que la gestión del conocimiento está ubicado en la ordenación, existencia y traspaso en su entorno, y potencializa el capital intelectual y crea capacidad en estos la institución, al hacer que el conocimiento sea utilizado por los integrantes sociales de una organización y reescrito de acuerdo con los cambios contextuales, por lo que la entidad tendrá que ser proactiva y pensar en el futuro (Montoya et al., 2018; Acevedo, 2017; Oberto y Oberto, 2016). Por lo antes mencionado, el objetivo del presente artículo ha sido: analizar las técnicas y prácticas respecto de la GS y desarrollo sostenible en América Latina y el Caribe (2011-2021), igualmente se plantea tres objetivos específicos el primero fue analizar los enfoques estratégicos metodológicos de las investigaciones seleccionadas. El segundo fue analizar los resultados obtenidos en los artículos revisados. El tercero respecto de las Conclusiones y objetivos de los artículos revisados.

\section{ESTRATEGIAS METODOLÓGICAS O MATERIALES Y MÉTODOS}

En la siguiente investigación, se realizó una revisión sistemática de artículos originales (Moher et al., 2014), la que permitieron sintetizar la información científica; así pues, en esta investigación el protocolo aplicó los ajustes expuestos para la revisión (Úsuga et al., 2020). El presente análisis se realizó sobre la "gestión social" en base a una búsqueda de artículos de investigación de los años 2011 a 2021 en Scielo, Ebsco, Dialnet y Redalyc. Con un total de 1,270 artículos ubicados; de estos se descartaron aquellos que no contaban con criterios de inclusión: estudios de desarrollo sostenible, estudios desarrollados en 
América Latina y el Caribe, investigaciones aplicadas, investigaciones publicadas en español y artículos de acceso abierto. A cerca de los criterios de exclusión se tuvo en cuenta a aquellos que hacían referencia a (García et al. 2017): estudios realizados de sustentabilidad y gobernabilidad, investigaciones aplicadas fuera del medio geográfico latinoamericano. Como resultado se obtuve un total de 10 artículos que cumplen con los objetivos propuestos, y son muy importantes para la presente revisión.

\section{Figura 1}

\section{Proceso de revisión PRISMA}

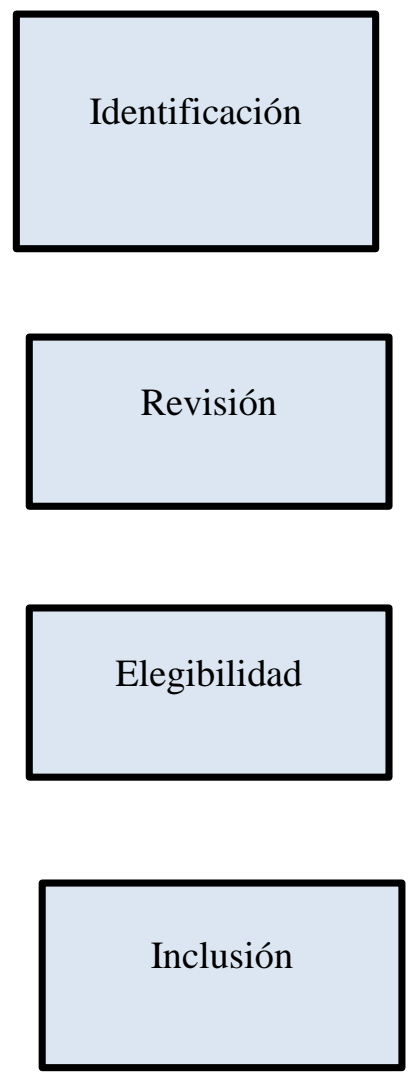

Artículos encontrados en base de datos (Redalyc, Ebsco, Scielo y Dialnet) todos ellos referidos a Gestión social entre el 2011 y 2021. n= 1,270

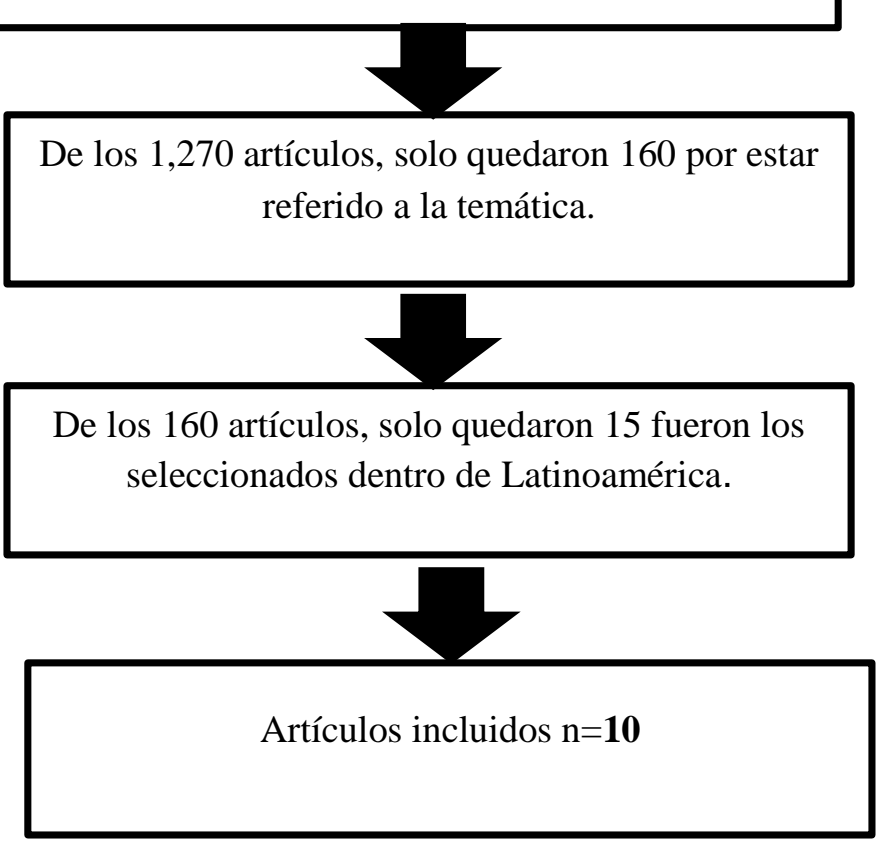

Nota: Se aclara que la depuración de los referidos en las bases de datos se realizó siguiente el modelo PRISMA.

Tabla 1 
Resultado de selección en los repositorios

\begin{tabular}{ccccccccc}
\hline Repositorio & Código & Combinación de búsqueda & Resultados & Temática & Región & Accceso & Objetivos & $\begin{array}{c}\text { Artículos } \\
\text { seleccionados }\end{array}$ \\
\hline EBSCO & A1 & "Gestión social" & 823 & 16 & 4 & 4 & 4 & 3 \\
SCIELO & B1 & "Gestión social" & 85 & 17 & 3 & 3 & 3 & 2 \\
DIALNET & C1 & "Gestión social" & 346 & 120 & 3 & 3 & 2 & 2 \\
REDALYC & D1 & "Gestión social" & 16 & 7 & 5 & 4 & 4 & 3 \\
& & Total & 1270 & 160 & 15 & 14 & 13 & 10 \\
\hline
\end{tabular}

En la siguiente tabla 1 se puede observar el proceso de selección, donde en función a los artículos elegidos y la razón de búsqueda (gestión social) se pudo obtener 1,270 artículos. No obstante, tomando en cuenta los estudios de desarrollo sostenible, estudios desarrollados en América Latina y el Caribe, y su relación con los objetivos se seleccionaron solo 10 investigaciones.

\section{RESULTADOS Y DISCUSIÓN}

En base a los objetivos formulados, se presentarán los resultados de las investigaciones de los 10 artículos encontrados.

\subsection{Características de los artículos}

Utilizando el proceso de revisión prisma, se puede observar que los diez (10) artículos seleccionados en la presente revisión (Serrano at al.,2021; Romero et al., 2020; Castaño y Arias, 2021; Altamirano et al., 2021), en su mayoría de investigaciones publicados en América Latina y el Caribe, respecto de la gestión social, se encuentran en Colombia, Ecuador, Cuba, Venezuela, México, Brasil, Panamá y Perú, de las cuales dos (2) investigaciones corresponden a Colombia sobre gestión social; luego esta con dos (2) trabajos publicados Ecuador, después Perú, Cuba, Venezuela, México, Brasil y Panamá, países en los que se ha realizado investigaciones por regiones.

\section{Tabla 2}


Artículos incluidos en la revisión

\begin{tabular}{ccc}
\hline Repositorio & Autor, año & País \\
\hline Ebsco & (Salamanca, 2016) & Panamá \\
Ebsco & (García et al., 2017) & Ecuador \\
Ebsco & (Muñoz, 2017) & Colombia \\
Scielo & (Rousseau, 2017) & México \\
Scielo & (Carbal y Blanquicett, 2011) & Colombia \\
Dialnet & (Ojeda y Rosas, 2015) & Cuba \\
Dialnet & (Chávez et al., 2019) & Venezuela \\
Redalyc & (Barba y Gavilánez, 2016) & Ecuador \\
Redalyc & (Ariel et al., 2019) & Brasil \\
Redalyc & (Bardales y Machuca, 2016) & Perú \\
\hline
\end{tabular}

Nota. La tabla 2, está referido a la bases de datos por autor y país.

\section{Figura 2}

Porcentaje de fuentes por país de procedencia

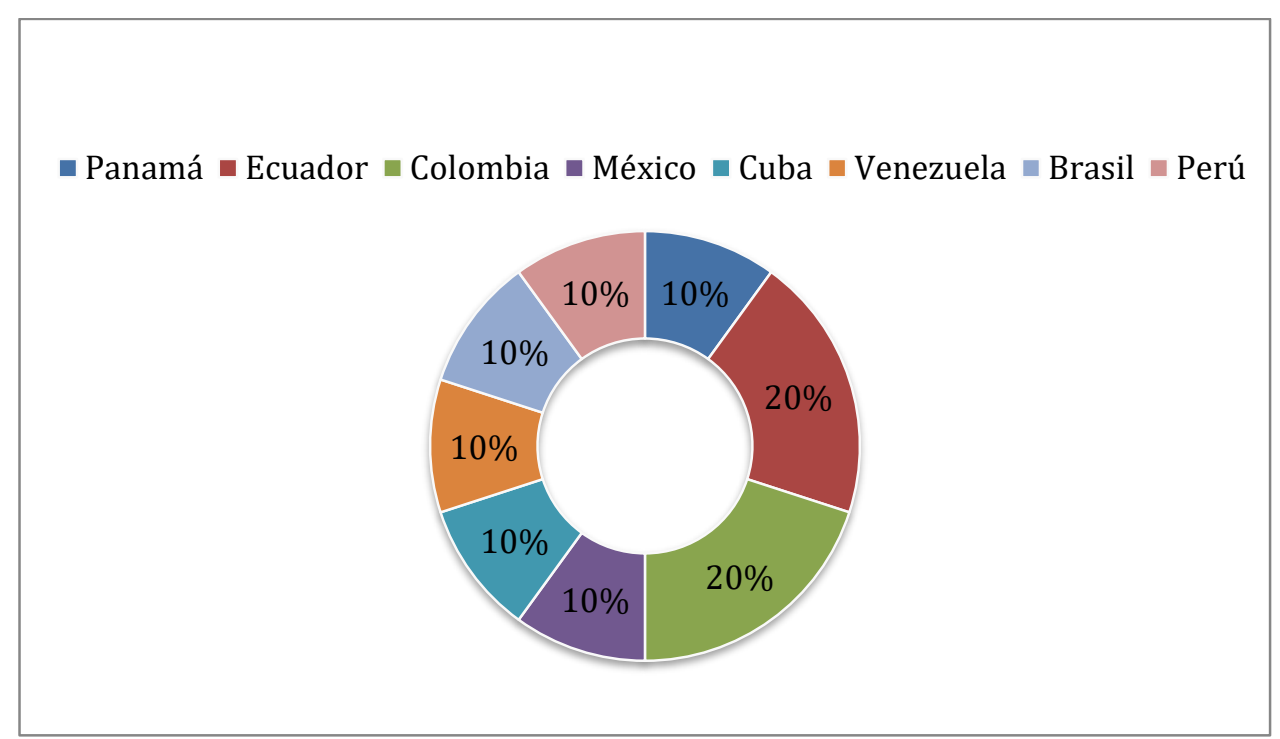

Nota: se especifica el origen de los artículos por países

\subsection{Estrategias y metodología empleada}

De la revisión realizada de las investigaciones no existe una preferencia respectos al enfoque metodológico. Como se puede observar que, de acuerdo a lo señalado por los autores, no existe una metodología relevante respecto de las otras. Consideramos que tres (3) de las investigaciones los autores eligieron el enfoque cuantitativo (Salamanca, 2016; García et al., 2017; Barba y Gavilánez, 2016), tres (3) optaron por un enfoque mixto (Chávez et al., 2019; Ariel et al., 2019; Bardales y Machuca, 2016) y por un lado 
Rousseau (2017) logra desarrollar una investigación cualitativo. Asimismo, tres (3) decidieron utilizar otras metodologías distintas a lo mencionado anteriormente.

De igual manera, sobre los instrumentos de recolección de datos, podemos decir de las investigaciones, cuatro (4) de estos eligieron la encuesta. De la misma manera, se puede apreciar que tres (3) investigaciones optaron por la entrevista, además, uno (1) opto por el instrumento de la observación y tres (3) optaron por otros instrumentos de recojo de datos (Carbal y Blanquicett, 2011; Ojeda y Rosas, 2015; Muñoz, 2017).

Tabla 3 Enfoque metodológico

\begin{tabular}{ccc}
\hline Autor, año & Enfoque & Instrumento de recolección de datos \\
\hline (Salamanca, 2016) & Cuantitativo & Encuesta (familias) \\
(García et al., 2017) & Cuantitativo & Encuesta (directivos) \\
(Muñoz, 2017) & Otros & Otros (poblaciones) \\
(Rousseau, 2017) & Cualitativo & Intrevista (empresas) \\
(Carbal y Blanquicett, 2011) & Otros & Otros (empresas) \\
(Ojeda y Rosas, 2015) & Otros & Otros (beneficiarios) \\
(Chávez et al., 2019) & Mixto & Observación (documentos) \\
(Barba y Gavilánez, 2016) & Cuantitativo & Encuesta (directivos) \\
(Ariel et al., 2019) & Mixto & Intrevista (representantes) \\
(Bardales y Machuca, 2016) & Mixto & Encuesta, Intrevista (familias) \\
\hline
\end{tabular}

Nota. La tabla 3, está referido a los autores, por enfoque que incluye también instrumentos de datos.

Figura 3 Referido al enfoque metodológico

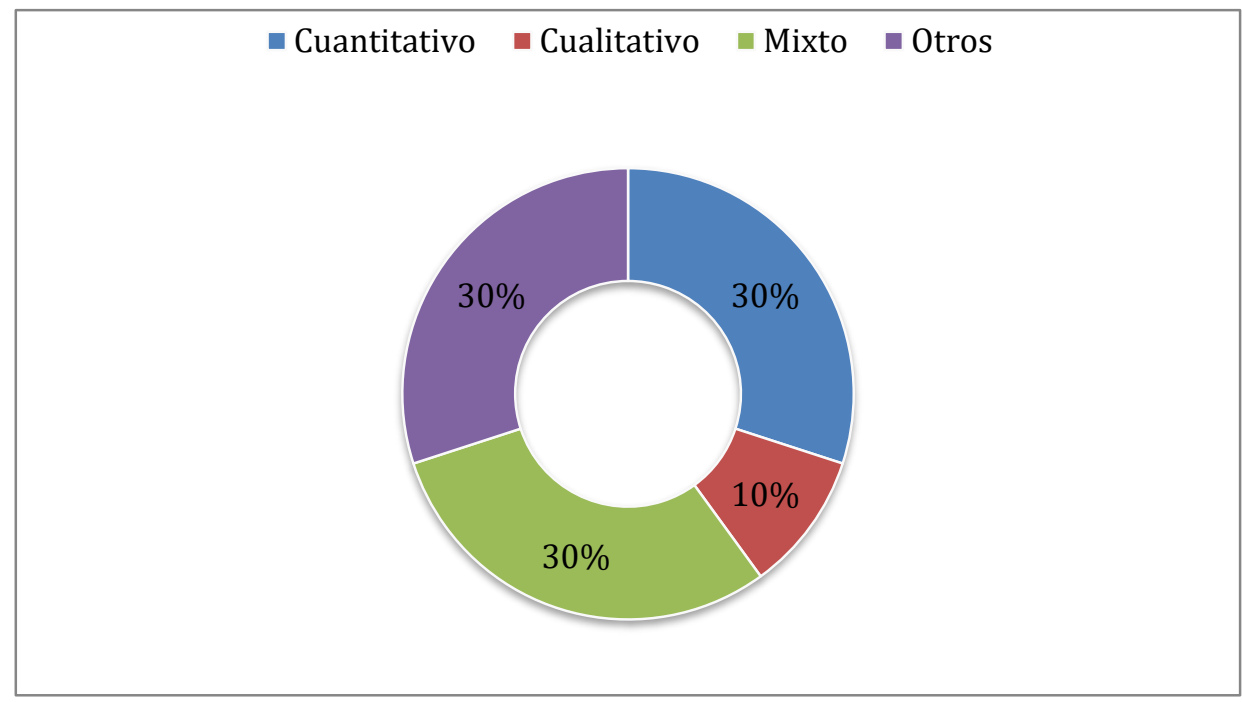

Nota. La figura 3, en la cual se incluye el enfoque a actores, representado en porcentaje. 


\section{Figura 4}

Instrumentos de recolección de datos

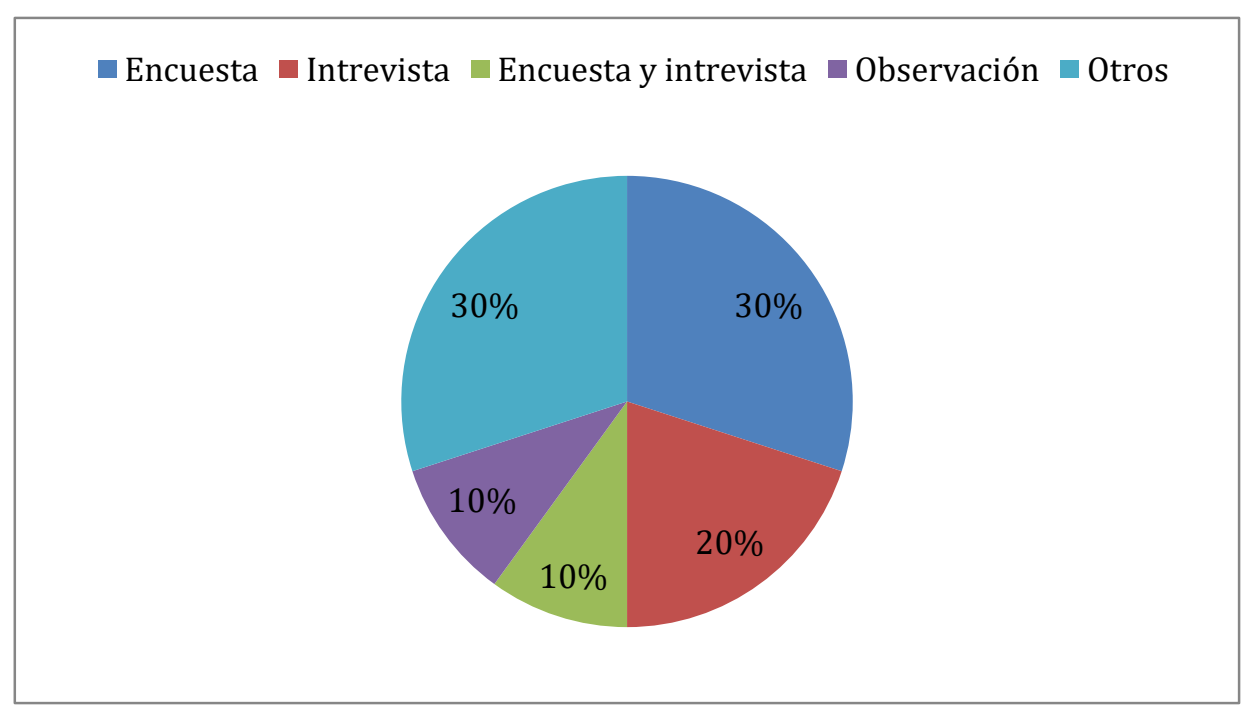

Nota. La figura 4, en la cual se incluye instrumentos de datos aplicado a actores, representado en porcentaje.

\section{Figura 5}

Cuestionario aplicado

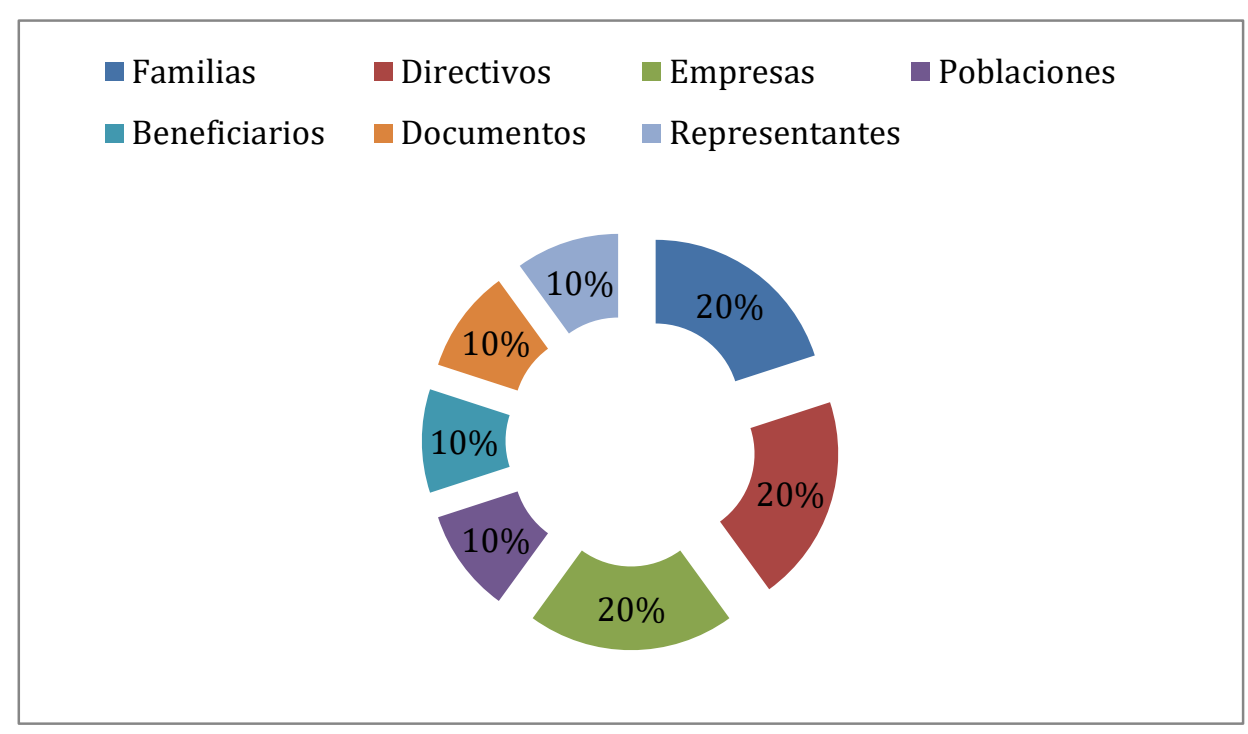

Nota. La figura 5, en la cual se incluye cuestionario aplicado a actores, representado en porcentaje.

Como se puede apreciar la figura la prioridad de realizar cuestionario al momento de recabar datos, el respectivo análisis presentado está en preferencia a las empresas, familias y directivos, siendo los actores importantes en la gestión social. También están 
las poblaciones, beneficiarios, documentos y representantes con una participación mínima.

\subsection{Conclusiones y objetivos de los artículos revisados}

Sobre la gestión social, de las 10 investigaciones analizadas, se pudo clasificar en 4 objetivos claros, en la cual la mayoría realizaron objetivos descriptivos. El primero es identificar estrategias que garanticen la sostenibilidad de una organización en el largo plazo, propiciar mayor desarrollo industrial y económico, así como mejor seguridad energética, promover el desarrollo sostenible mediante la difusión y la reaplicación a escala de Tecnologías Sociales (TS); y aportar a la creación de las condiciones de sostenibilidad ambiental. Como segundo se habla mucho de describir y diseñar saberes, y productos en una comunidad. Tercero conseguir el pleno desarrollo de las personas, construir un modelo de convivencia urbana, a partir de generar un cambio cultural desde el hábitat de la población vulnerable de los proyectos de vivienda. Finalmente tenemos el cuarto que es evaluar, de acuerdo con estudios descriptivos y correlacionales, la gestión social de las Cooperativas de Ahorro y Crédito.

\section{CONCLUSIÓN O CONSIDERACIONES FINALES}

El objetivo de este estudio fue analizar las técnicas y prácticas respecto de la Gestión Social y desarrollo sostenible en América Latina y el Caribe una revisión sistemática. Es importante manifestar que la escasez de publicaciones científicas sobre el tema dificulta generalizar los resultados y beneficios de Gestión Social en el bienestar que son explicados en los textos analizados. Es así cuando motivan la realización de nuevas investigaciones que permitan validar la eficacia de este prometedor método en las áreas de las empresas. Por una parte, el guía de mejora para nuevos estudios de investigación deberá contener el uso de colectividad en el control, la aplicación de enfoques en las distintas investigaciones.

Este contenido supone el punto de partida para crear conocimiento científico sobre esta técnica en lo que viene siendo denominado "gestión social" potencialmente un importante instrumento de trabajo comunitario y garanticen la sostenibilidad de una organización.

De acuerdo a los resultados se pueden extraer lo siguiente: sabiendo que Ecuador es el país donde se concentra la mayor cantidad de investigaciones sobre enfoque cuantitativo y encuestas aplicados a directivos, y seguido de Colombia donde se encuentra en un 
estado más preliminar de desarrollo; el instrumento más utilizado para medir es la encuesta con adaptaciones para su uso en diferentes contextos.

\section{LISTA DE REFERENCIAS}

Ariel B., Guillaumon S. y Cirilo M. (2019). Redes interorganizacionales, gestión social y desarrollo territorial: el caso de la Red de Tecnología Social, Brasil. Pampa. Revista Interuniversitaria , 120-138. DOI: 10.14409/pampa.2019.20.e0014.

Altamirano C., Castillo E. y Rodríguez I. (2021). Biodanza: revisión sistemática sobre los beneficios de una práctica emergente en la promoción de la salud y el bienestar. Retos, 844-848. http://hdl.handle.net/10272/19003.

Abdelfattah L. (2018). Different Typologies of Social Management in the Context of Changing Social Housing Service Schemes. researchgate, 1-10. https://www.researchgate.net/publication/333668427.

Acevedo S. (2017). Gestión social del conocimiento, redes de investigación e innovación para la inclusión . Revista Científica Electrónica de Ciencias Gerenciales, 62-73. http://www.redalyc.org/articulo.oa?id=78252811005.

Barba D. y Gavilánez M. (2016). Gestión social en las Cooperativas de Ahorro y Crédito de una provincia ecuatoriana. Revista Ciencia, 9(19), UNEMI, 30-38. https://www.redalyc.org/articulo.oa?id=582661268003.

Bardales J. y Machuca G. (2016). Estudio de la gestión social y productiva local de la actividad agrícola en la comunidad de Yanallpa, Requena, Perú. Apunt. cienc. soc., 06(02), 118-130. http://dx.doi.org/10.18259/acs.2016020.

BID. (2017.). Manual de gestión social. https://publications.iadb.org/publications/spanish/document/Manual-degesti\%C3\%B3n-social.pdf.

Carbal A. y Blanquicett J. (2011). Sistema de gestión social para pequeñas y medianasempresas. Saber, Ciencia y Libertad, 6(1), 105-118. https://dialnet.unirioja.es/servlet/articulo?codigo $=5755298$.

Castaño A. y Arias S. (2021). Efecto de la responsabilidad social corporativa en la reputaciónde las organizaciones: na revisión sistemática. Revista Universidad \& Empresa, 23(40), 1-25. https://doi.org/10.12804/revistas.urosario.edu.co/empresa/a.8859. 
Chávez S., Pérez V. y Barrientos N. (2019). Gestión social en empresas de consumo $\begin{array}{lll}\text { masivo. } & \text { Cicag, } & \text { 55-75. }\end{array}$ https://dialnet.unirioja.es/servlet/articulo?codigo $=6844338$.

García G., Sánchez V., Elizalde D. y Fernández A. (2017). La gestión social de Cooperativas de Ahorro y Crédito en Ecuador. Revista de Cooperativismo y Desarrollo, $\quad 5(1), \quad 47-59$. http://coodes.upr.edu.cu/index.php/coodes/article/view/152.

García I. Mora J., Estrada J. y Piñeros R. (2017). ¿Cuál es el Efecto de la Moringa oleiferasobre la Dinámica Ruminal? Revisión sistemática. Rev Inv Vet Perú, 4355 .http://dx.doi.org/10.15381/rivep.v28i1.11675

Hernández L. (2017). Rediseño Curricular de la Carrera Gestión Social y Desarrollo de la Universidad de Otavalo para su Proceso de Acreditación. Formación Universitaria, 3-16. http://dx.doi.org/10.4067/S0718-50062017000600002 .

Mendieta L., Vargas T., Barros R. y Páez H. (2018). Evaluación del nivel de satisfacción de la gestión social del conocimiento (GSC) de la Universidad de Guayaquil. Revista Arjé. 12(22), 432-446. http://arje.bc.uc.edu.ve/arje22/art51.pdf.

Miyazaki M., Oxilia V. y Leiva M. (2020). La educación ambiental relacionada al desarrollo sostenible en las instituciones educativas del municipio de Villa Hayes. $\begin{array}{lllll}\text { Reportes científicos la } & \text { de }\end{array}$ https://doi.org/10.18004/rcfacen2020.11.02.14.

Moher D., Liberati A., Tetzlaff J. y Altman D. (2014). Ítems de referencia para publicar Revisiones Sistemáticas y Metaanálisis: La Declaración PRISMA. Rev Esp Nutr Hum Diet., 172-181. http://creativecommons.org/licenses/by-ncnd/3.0/deed.es_CO.

Montoya L., Arenas D. y Di Lorenzo S. (2018). Gestión social del conocimiento y análisis prospectivo de su incidencia en la universidad contemporánea. MEDISAN, 449459. http://scielo.sld.cu/pdf/san/v22n4/san15224.pdf.

Moreno G., Dávila M. y Arboleda R. (2019). Gestión social y desarrollo comunitario: caso de la comunidad negra Campo Hermoso, Buenaventura, Pacífico $\begin{array}{llll}\text { colombiano. } & \text { Revista } & \text { Científica } & \text { Sabia, }\end{array}$ https://doi.org/10.47366/sabia.v5n1a9. 
Muñoz G. (2017). Las técnicas de la "gestión social” de poblaciones impactadas por proyectos hidroeléctricos como instrumento de gobierno. AGO.USB, 17(2), 324613. https://orcid.org/0000-0001-6142-0605 .

Oberto A. y Oberto G. (2016). Gestión social del conocimiento y aprendizaje ubicuo como estrategia. Serbiluz, 373-390. https://dialnet.unirioja.es/servlet/articulo?codigo=5901097.

OCDE. (2016). Evaluaciones del desempeño ambiental Perú. https://www.oecd.org/environment/country-reviews/1600313\%20Evaluacion\%20desempeno-Peru-WEB.pdf

Ojeda L. y Rosas R. (2015). Aplicación de la auditoría de gestión social cooperativa en la cooperativa de producción agropecuaria camilo cienfuegos. Revista Cooperativismo $\quad y \quad$ Desarrollo, $\quad 3(1), \quad 1-13$. https://dialnet.unirioja.es/servlet/articulo?codigo=5233959.

Quintana C., Alum N. y Monterey C. (2018). La gestión social para fomentar los valores en la prevención del delito y la indisciplinas sociales . Avances, 27-39. http://www.ciget.pinar.cu/ojs/index.php/publicaciones/article/view/310.

Romero D., Pertuz V. y Orozco E. (2020). Factores determinantes de competitividad e integración organizacional: revisión sistemática exploratoria. Información Tecnológica, 31(5), 21-32. http://dx.doi.org/10.4067/S071807642020000500021 .

Rousseau I. (2017). La nueva regulación de la gestión social de los proyectos energéticos en México. Seguridad, sustentabilidad y gobernabilidad. Revista Mexicana de Ciencias Políticas y Sociales, 62(230), 197-220. https://doi.org/10.1016/S01851918(17)30021-1.

Salamanca L. (2016). La gestión social en proyectos de vivienda de interés social. Cuadernos de Vivienda y Urbanismo, 9(18), 308-325. http://dx.doi.org/10.11144/Javeriana.cvu9-18.gspv.

Serrano M., Zamanillo R., Castro A., Fiol M. y Ricci I. (2021). Insomnio y calidad del sueño en profesionales sanitarios frente a la Covid-19: una revisión sistemática de la literatura y meta-análisis. Actas Esp Psiquiatr, 49(4), 155-179. http://eds.a.ebscohost.com/eds/detail/detail?vid=0\&sid=c377603f-49bd-4a618392-a507d9c4ce1c\%40sdc-v- 
sessmgr03\&bdata=Jmxhbmc9ZXMmc210ZT11ZHMtbGl2ZQ\%3d\%3d\#AN=151 $201934 \& d b=$ fua.

Terziev V. (2018). Active social programs development in bulgaria: contemporary challenges and social management instruments. Ijasos, 597-611. http://ijasos.ocerintjournals.org.

Úsuga F., Higuita L. y Cardona J. (2020). Revisión sistemática de las aplicaciones de la escala CS-DS Chronic Self Destructiveness Scale, 1985 - 2019. 1-26. https://rcientificas.uninorte.edu.co/index.php/psicologia/article/view/12798/2144 21445152.

Varona R. (2018). Acciones comunitarias para la gestión social del conocimiento medio ambiental en la comunidad Edecio Pérez en el municipio de Holguín. Rev. gen. inf. doc, 477-488. http://dx.doi.org/10.5209/RGID.60805.

Zacarias A., Canto R. y Méndez F. (2020). Bases para el diseño de políticas públicas de manejo de recursos naturales vinculados al desarrollo sostenible, en la ciudad de Itauguá. Reportes científicos de la FACEN, 27-32. https://doi.org/10.18004/rcfacen2020.11.02.27. 